\title{
Simulative Study for the Impact of Signal Frequency on Precision of Grounding Wire Detection in 10kV Distribution Network
}

\author{
Jun $\mathrm{Lu}^{1, \text { a }}$, Lefeng Cheng ${ }^{2, \mathrm{~b}^{*}}$, Tao $\mathrm{Yu}^{2, \mathrm{c}}$ \\ 'Zhaoqing Power Supply Bureau, Zhaoqing, 526060, China \\ ${ }^{2}$ Electric Power College, South China University of Technology, Guangzhou, 510640, China \\ alujun_zhaoqing11@163.com, 'lefengg@126.com, 'taoyu1@scut.edu.cn
}

Keywords: electromagnetism simulation; PSCAD/EMTDC; grounding wire; detection; signal injection

Abstract. Aimed at temporary grounding wire detection problems in distribution network, some factors influence the detection precision, on this basis, an electromagnetic transient simulation model for temporary grounding wire detection was established based on PSCAD/EMTDC platform, the model simulates the actual process of grounding wire detection work. In the simulation model, the impact of signal frequency on the precision of grounding wire detection in distribution maintenance lines was studied. The simulation results show that different frequency of injection signal has impacts on the grounding wire detection due to the distributed capacitors and lines inductances. Based on simulative analysis, a medium frequency range, especially the inter-harmonics, were suitable for signal injection detection. Meanwhile a threshold current method was proposed as detection criterion, and the method was simple and practical.

\section{Introduction}

In actual distribution lines maintenance or rebuilding work, hanging the grounding wire is an important part, and during the work, the work ticket or telephone is used to contact the power dispatching department to determine the transmission lines in outage or the equipment, according to the requirements, the department sends the commands to the operators to implement switch operation and grounding isolators closing, and at the same time the operators fill out the operation ticket according to the commands and finish operation dispatch. After the operators receiving the instruction to work, the grounding wire is hanged according to the actual construction situation, and the workers can start work on the transmission lines or equipment, and after the completion of the work, all safety measures should be removed and the workers report to the power dispatch and the power system recovers the power supply. The maintenance work is mainly performed based on operating ticket system and operating degree of operation procedures by field operators, thus there is a vulnerability and possibility of misoperation ${ }^{[1-4]}$. It is well know that there are multi points and extensive aspects during the maintenance or rebuilding work in distribution network, and the occurrence probability of missing removing the grounding wires or artificially setting short lines in malice. Therefore, the accident of closing the power with a grounding wire may happen, that is, the operators recover the power supply under the situation of missing removing grounding wire or pulling the grounding isolator. According the statistics in literature [4], the occurrence of grounding accident was still frequent in $110 \mathrm{kV}$ distribution network and under the voltage level since the year of 2003 2009, and which accounting for about $25 \%$ of all kinds of malignant accidents. Obviously, only rely on the operating system, such misoperation accidents cannot completely be avoid in recent years, and with the rapid increase in the size of the grid, such kind of accident will further increase in further.

Several grounding points detectors or monitoring systems appear in high voltage transmission network and substations in recent years. In $110 \mathrm{kV}$ and above the voltage level transmission network, the detection precision for grounding points is relatively higher, while in low voltage network the detectors seldom appear. In aspects of grounding wire detection methods in distribution network, the literature [5] proposed a novel single-phase ground fault location algorithm for series compensated line based on improved RL model, and it is well considered of the effect of non-linear MOV and 
compensated capacitor on actual reactance of transmission line. A fault line selection method of distribution network based on improved Hilbert-Huang Transform and identification confidence degree was proposed in literature [6], in which the zero sequence current of each line can be decomposed by applying the improved HHT and the instantaneous phase angle and amplitude information of fault feature point were obtained, and fault line selection voting mechanisms were got by comparing phase and angle relationship, the proposed approach has high accuracy, shorten calculation time, and is not affected by the fault position, initial fault phase, and earth resistance. A transmission line temporary ground wires detection device based on traps was designed in literature [7], which was exclusive for the detection of temporary ground wires connected to the ground through towers, and added sinusoidal signal of $200 \mathrm{kHz}$ to power outage line and could decide whether there is any ground wires attached to the power line according to the amplitude of $200 \mathrm{kHz}$ signal of the sampling point. The designed device was taken with a $200 \mathrm{kV}$ overhead transmission line in Hebei Province and the result provided that it can effectively identify ground wires grounded to the earth through intermediate portion of the tower, that is to say, the result verified the validity of the device. A novel accurate location of faults on power transmission line with two-end phasor measurement used in differential protection device was proposed ${ }^{[8]}$, and the new algorithm may solve the shortages such as complex calculations of calculating the hyperbolic functions, large amount of calculation with the Newton-Raphson iteration and binary search and needing accurate line parameters. In other studies, an accurate fault location algorithm for transmission lines based on R-L model parameter identification was proposed in literature [9] and a new two-terminal numerical algorithm for fault location, distance protection, and arcing fault recognition was studied in literature [10].

Based on these studies, it is concluded that the researches on grounding wire detection in $10 \mathrm{kV}$ and bellow the voltage level distribution network are seldom, thus it's significant to design smart detectors for grounding points detection in low voltage distribution network and aimed at the complexity of distribution lines maintenance, it's need to take into consideration of the influences on the detection precision of grounding wires. Therefore, based on signal injection detection scheme used in distribution network, this paper mainly simulated the impacts of frequency of injective signal on the precision detection of grounding wires. The simulative results shown that the impact should be taken into consideration, due to the distributed capacitors and according to the simulation analysis, a threshold current method as detection criteria was proposed, and the method was simple and practical.

\section{Electromagnetism simulation model establishment}

Based on early field research and theoretical analysis, a kind of electromagnetism simulation model based on PSCAD/EMTDC software platform was established, which was used for grounding wire detection. The simple simulative model was shown in Fig.1, in which a group of grounding wire was injected detection signal into the model, and the other group of grounding wire was used to control the switch to simulate the existence of grounding wire or not, and the judgment was on basis of the current in the grounding wire. The simulative time was set $0.5 \mathrm{~s}$, the grounding wire was connected to distribution line in beginning, and which was removed at the time of $0.25 \mathrm{~s}$, the two cases of existence and no existence of grounding wire can be compared in the same current simulation wave diagram. A resistance was a substitute for the earth resistance, and the specified value was took $4 \Omega$, and the influence of different size of grounding resistance was studied in next. The length of actual maintenance line had a significant variation, some were multi-hundred miters, and some were even tens of kilometers, in the simulative model, the length of transmission line was supposed as $5 \mathrm{~km}$, and the distance of two groups of grounding wires was $3 \mathrm{~km}$. In order to simulate the actual physical environment of hanged grounding wire more accurately, the simulation software of PSCAD/EMTDC was adopted to perform correlational studies, and a suitable frequency of injection signal was selected to validate validity of proposed detection schemed in post paper.

The PSCAD software was a power system simulator based on graph, which was a impactful graphical user interface (GUI), and can effectively improve the simulative research efficiency of 
electromagnetic transient of power system; EMTDC was an electromagnetic transient simulative program based on software, and could be called by the PSCAD, the wonderful function of EMTDC was its simple simulation for complex power system, including DC transmission system and other control system ${ }^{[11]}$.

Based on the analysis, the model of overhead line was selected the frequency dependent (phase) model (FDM), which was the most advanced and accurate time domain analysis model of transmission line. The FDM was need to set the parameters of tower and conductors which including the conductor radius and resistance and so on. The concrete parameters for simulation building were shown as follows.

The minimum distance of conductor was related to the span in distribution network, the span was took 40 50 meters in town, and 60 100 meters in suburban district. Generally, the span was took 80 meters in most suburbs and villages, the minimum wire spacing was took 0.85 meter, and it was selected 1 meter when in simulation and the conductors were horizontally-arranged. While the height of tower wasn't generally immovable and which was selected 15 meters in the simulative model, the type selection interface of tower was shown in Fig.2, and a complex simulation model for grounding wire detection in distribution was shown in Fig. 3. During the simulation, the grounding wire was replaced by a grounding resistance, and its specified value was no larger than $4 \Omega$, and was took $2 \Omega$ when in actual simulation.

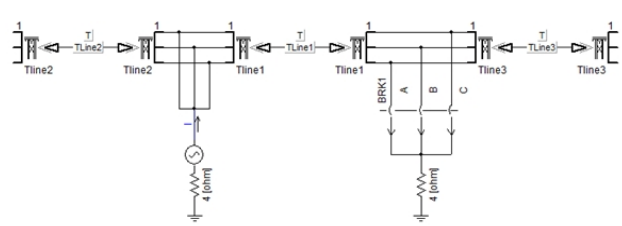

Fig.1 A simple simulative model

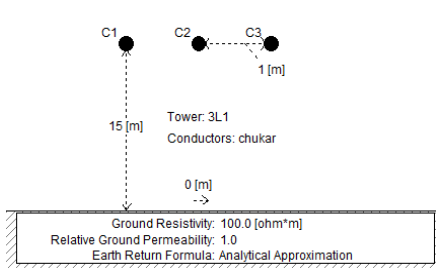

Fig. 2 Selection of tower

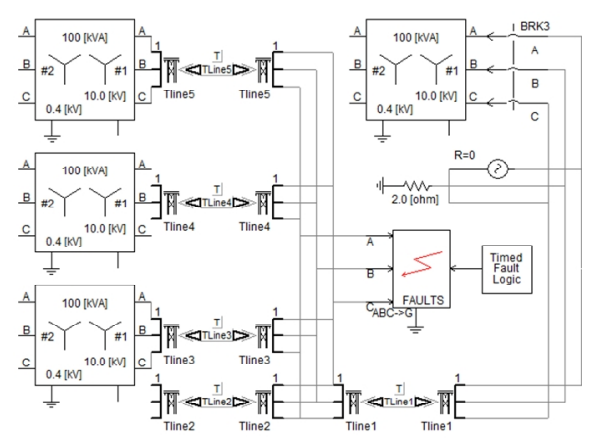

Fig. 3 A complex simulative model

\section{Simulative analysis}

Simulation for different frequency of signal injection. Based on the aforementioned simulative model, under different frequency of signal injection, the current results of simulation was shown in Fig.4 (a) (h), in the simulation process, suppose in $0 \sim 0.25 \mathrm{~s}$ the grounding wire was existent, and was removed after $0.25 \mathrm{~s}$.

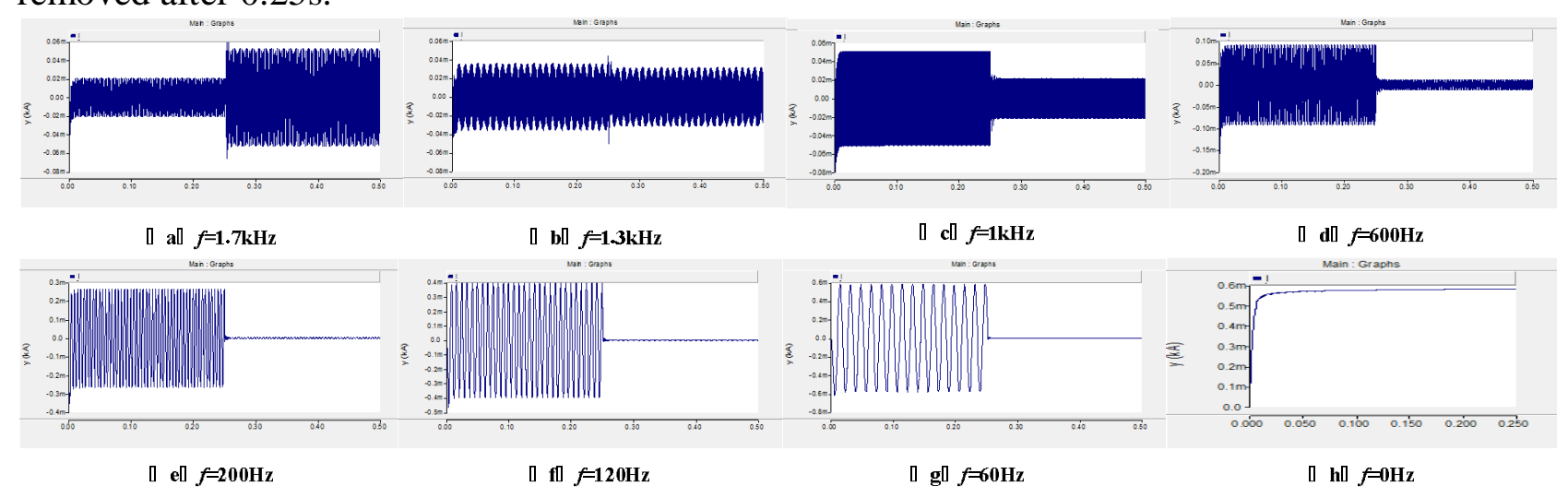

Fig.4 The detecting current in different frequency injection

Seen from Fig.4, we find that the higher the frequency of signal injection, the smaller of the difference of current measured under the case of whether other grounding wires exist or not, and even the current when no other grounding wires exist is larger than when other grounding wires exist. This is due to the existence of distributed capacitor whose capacitive reactance is related to frequency and the higher the frequency, the larger of the reactance value, and meanwhile, the smaller of the impedance, 
and when the total capacitive reactance is smaller than grounding resistance, then the case that the current when no grounding wires exist is larger than the existence of grounding wires will happen. Based on the analysis, the frequency of signal should be low, and if the frequency is too small, the induced electromotive force will become small; and on other hand, the injective signal may be influenced by the disturbance of field $50 \mathrm{~Hz}$ of power frequency and harmonic, which are should be filtered in the secondary circuit, and for a convenient filter, the frequency of injective signal should be selected between $n$ times and $n+1$ times of harmonic, among it, $n$ is an integral number. Theoretically, $n$ could be selected any value, but if the value is selected larger, on one hand, the value of distributed inductance increases with the signal frequency, on other hand, the capacitance of line increases with the decrease of signal frequency, both the inductance and capacitance have an impact on the existence judgment of grounding wire. It is known that the value of distributed capacitance is not only related to the frequency of signal injection but also the length of transmission line, the longer the length, the smaller the value. Thus the frequency of injective signal should be selected suitable under considering the most extreme case. The length of line is supposed $80 \mathrm{~km}$ in simulation, and different frequency of injective signal was simulated, the simulation results are shown in Tab. 1.

Tab.1 Simulative results of grounding wire detection in $80 \mathrm{~km}$ transmission line under different frequency of signal injection

\begin{tabular}{ccc}
\hline \multirow{2}{*}{ Frequency of injective signal $(\mathrm{Hz})$} & \multicolumn{2}{c}{ The effectvie value of current in grounding wire (A) } \\
\cline { 2 - 3 } & Other grounding wires exist & No grounding wires exist \\
\hline 60 & 0.0915 & 0.0023 \\
\hline 120 & 0.0500 & 0.0046 \\
\hline 180 & 0.0326 & 0.0071 \\
\hline 240 & 0.0235 & 0.0099 \\
\hline 310 & 0.0167 & 0.0138 \\
\hline
\end{tabular}

Threshold current method. According to Tab.1 above, the effective value of current in the grounding wire is directly related to the frequency of injective signal especially when other grounding wires exist, meanwhile in the case that no grounding wires exist, the effective value of current is generally small, which is about several mAs to several tens of mAs. When considering a certain margin, thus a suitable medium frequency range of injective signal was choosen to detect the grounding wire in this paper. The judging algorithm to judge whether a group of grounding wire exists is based on the threshold curret method, generally, even-order harmonic voltage doesn't exist in the transimission line, so we can choose the even-order harmonic as injectvie signal, certainly, if we choose the inter harmonic, the effect will be better, the disturbance is smaller in that way, the criteria is set as follows:

$$
Q_{y u} \geq \chi \cdot Q_{c e}
$$

Where $Q_{\text {yu }}$ is threshold value, which is generally smaller than $10 \mathrm{~mA}$, can take $5 \mathrm{~mA}$ or $6 \mathrm{~mA}$, etc., and it can be adjusted according to the actual field situation, $Q_{\mathrm{ce}}$ is effective value of current in detection loop circuit; $\chi$ is correction factor; $Q_{\mathrm{yu}}=U_{\text {in }} / Z$ and $U_{\text {in }}$ is effevtive value of actual injective signal; $Z$ is setting impedance range, generally takes $1 \mathrm{k} \Omega$, when the detection condition meets the formula (1), that is, when the value of detection signal is lower than the threshold value, we can judge that on temporary hanged grounding wires exist in current maintenance or rebuilding line, otherwise, at least a group of grounding wire exists.

\section{Summary}

(1) The thesis is based on field investigation, and aimed at temporary hanged grounding wire detection problems in $10 \mathrm{kV}$ distribution network, and an electromagnetic transient simulation model which is suitable for regional power grid substations and transmission lines is established based on PSCAD/EMTDC. The model can simulate the physical environment of hanged grounding wire in situation of lines maintenance, and the model verifies the rationality of the proposed voltage signal injection detection scheme, and the proposed scheme is practical.

(2) The PSCAD/EMTDC simulation model is established to analyze the impacts of the frequency of injective signal on the precision of grounding wire detection, and the simulative results show that select 
a medium frequency range of voltage as injective signal (especially the inter-harmonics) is better. Based on simulative analysis, the threshold current method as detection criteria was proposed, the method was simple and in which, only the detection current in the loop is larger than the threshold value, we can judge at least one group of grounding wire exists.

\section{References}

[1] Jiajun Liu, Jun Miao, Lixiao Yao, et al, Visualized monitoring of grounding wire connection status for electric power maintenance operation, J. Electric Power Automation Equipment (in Chinese), 2010, 38(7): 134-136.

[2] Jiajun Liu, Bo Liu, Xin Xu, et al, Research on the grounding line information-gathering device of catenary operation, J. Power System Protection and Control (in Chinese), 2011, 39(5): 139-143.

[3] Jiajun Liu, Jun Miao, Research on new type of electric power overhaul grounding line device, J. Power System Protection and Control, 2009, 37(23): 119-121.

[4] Zhe Li, Dingzhu Zhong, Guanghua Li, et al, 100 cases of electrical misoperation accidents and its cause analysis, in: Beijing, China Electric Power Press, 2009.

[5] Jinhu Zhang, Zhenyu Xu, Qixun Yang, et al, A novel single-phase ground fault location algorithm for series compensated line based on improved RL model, J. Power System Protection and Control(in Chinese), 2015, 43(10): 1-7.

[6] Zhiling Ren, Yuanyuan Zhang, Fault line selection of distribution network based on improved Hilbert-Huang Transform and identification confidence degree, J. Power System Protection and Control(in Chinese), 2015, 43(10): 8-13.

[7] Qian Zhang, Feilong Wang, Yanqing Li, Design of transmission line temporary ground wires detection device based on traps, J. Inner Mongolia Electric Power(in Chinese), 2013, 31(2): 67-71.

[8] Liping Wang, Xiaoru Wang, Wei Wang, et al, Study of faults location utilizing algorithm on power transmission lines, J. Power System Protection and Control(in Chinese), 2014, 42(16): 52-58.

[9] Jiale Suonan, Jun Qi, Fufeng Chen, et al, An accurate fault location algorithm for transmission lines based on R-L model parameter identification, J. Proceedings of the CSEE(in Chinese), 2004, 24(12): 119-125.

[10]LEE C J, PARK J B, SHIN J R, et al, A new tow-terminal numerical algorithm for fault location, distance protection, and arcing fault recognition, J. Sci. IEEE Trans on Power Delivery, 2006, 21(3): 1460-1462.

[11] Tao Yu, Wenjia Ye, Haihua Liang, EMTDC simulation of outage line closing for transmission circuits lines parallel connection on single tower, J. Power System Protection and Control (in Chinese), 2010, 38(23): 159-163. 